\title{
Applications of the double and the dual numbers. The Bianchi models
}

\author{
G.F. Torres del Castillo \\ Instituto de Ciencias, Universidad Autónoma de Puebla, \\ 72570 Puebla, Pue., México.
}

Received 21 February 2020; accepted 24 February 2020

\begin{abstract}
We show that by using complex, double, and dual numbers one can find the invariant one-forms employed in the metrics of the Bianchi cosmological models. The result is equivalent to find, locally, all the Lie groups of dimension three.
\end{abstract}

Keywords: Double numbers; dual numbers; cosmology; Lie groups.

Mostramos que usando números complejos, dobles, y duales uno puede hallar las uno-formas invariantes empleadas en las métricas de los modelos cosmológicos de Bianchi. El resultado es equivalente a hallar, localmente, todos los grupos de Lie de dimensión tres.

Descriptores: Números dobles; números duales; cosmología; grupos de Lie.

PACS: $02.20 . \mathrm{Qs} ; 98.80 . J \mathrm{k}$

DOI: https://doi.org/10.31349/RevMexFisE.17.146

\section{Introduction}

The double and the dual numbers can be regarded as generalizations of the complex numbers. The double numbers are objects of the form $a+\mathrm{j} b$, where $a$ and $b$ are real numbers, and $\mathrm{j}^{2}=1$ (but $\mathrm{j} \neq \pm 1$ ), while the dual numbers have the form $a+\varepsilon b$, where $a$ and $b$ are real numbers, with $\varepsilon^{2}=0$ (but $\varepsilon \neq 0$ ). As shown in Ref. [1], even though these numbers do not have all the algebraic properties of the real and the complex numbers, they are useful in the solution of the differential equations that appear in some problems of classical mechanics. These three sets of numbers are also useful to express the nonequivalent actions of $\mathrm{SL}(2, \mathbb{R})$ on the Cartesian plane [2].

In this paper we show that the complex, double, and dual numbers can be employed to find the invariant one-forms employed in the metrics of the Bianchi cosmological models or, equivalently, in finding locally all the Lie groups of dimension three, without making use of the exponential map. In Sec. 2, we summarize the algebraic properties of the double, and the dual numbers to be employed in the rest of the paper. In Sec. 3, with the aid of the formalism of differential forms, we show that the complex, double and dual numbers are very useful in finding the metric tensor for the cosmological Bianchi models or, equivalently, the Lie groups of dimension three.

\section{Algebraic preliminaries}

In the following section we shall make use of "hypercomplex" numbers of the form $a+\mathrm{h} b$, where $a$ and $b$ are real numbers and the unit $\mathrm{h}$ may be $\mathrm{i}, \mathrm{j}$, or $\varepsilon$. By definition, the hypercomplex numbers $a+\mathrm{h} b$ and $c+\mathrm{h} d$ are equal to each other if and only if $a=c$ and $b=d$. The real numbers $a$ and $b$ will be called the real and imaginary parts of $a+\mathrm{h} b$, respectively. The sum and the product of these numbers are defined in the natural manner

$$
(a+\mathrm{h} b)+(c+\mathrm{h} d)=a+c+\mathrm{h}(b+d),
$$

and

$$
(a+\mathrm{h} b)(c+\mathrm{h} d)=a c+\mathrm{h}^{2} b d+\mathrm{h}(a d+b c) .
$$

Then one can show that the sum and the product are commutative and associative, and that the product is distributive over the sum.

If $x+\mathrm{h} y$ is the multiplicative inverse of $a+\mathrm{h} b \neq 0$, then we must have

$$
\begin{aligned}
a x+\mathrm{h}^{2} b y & =1, \\
b x+a y & =0 .
\end{aligned}
$$

Hence, $a+\mathrm{h} b$ possesses a multiplicative inverse if and only if $\Delta \equiv a^{2}-\mathrm{h}^{2} b^{2}$ is different from zero. Thus, the double number $a+\mathrm{j} b$ does not possess a multiplicative inverse if $a= \pm b$, and the dual number $a+\varepsilon b$ does not possess a multiplicative inverse if $a=0$. This implies that the double and the dual numbers are not fields with the operations defined above, but only rings; however, this fact does not limit their usefulness for some purposes.

\section{The Bianchi models}

In the Bianchi models of the relativistic cosmology, it is assumed that the space-time is foliated by three-dimensional hypersurfaces with a metric admitting a three-dimensional isometry group that acts transitively on these hypersurfaces (see, e.g., Refs. [3-6]). This means that the metric tensor of each of these three-dimensional hypersurfaces possesses three Killing vectors, which are linearly independent at each point, and generate a Lie algebra. If $\left\{\mathbf{X}_{1}, \mathbf{X}_{2}, \mathbf{X}_{3}\right\}$ is a basis for these Killing vectors, then $\left[\mathbf{X}_{i}, \mathbf{X}_{j}\right]=c_{i j}^{k} \mathbf{X}_{k}$, where the $c_{i j}^{k}$ are constants, the Latin indices $i, j, k, \ldots$ run from 1 to 3 , and there is summation over repeated indices. The structure constants have to satisfy the relations

$$
c_{i j}^{k}=-c_{j i}^{k}, \quad c_{i j}^{m} c_{m k}^{l}+c_{j k}^{m} c_{m i}^{l}+c_{k i}^{m} c_{m j}^{l}=0 .
$$


All the solutions of these conditions can be given explicitly and constitute the various Bianchi types [3-6].

The metric of the hypersurfaces mentioned above is of the form $a_{i j} \omega^{i} \otimes \omega^{j}$, where the $a_{i j}$ are the entries of a symmetric positive definite matrix, constants on each hypersurface, and the one-forms $\omega^{i}$ are invariant under the transformations generated by the vector fields $\mathbf{X}_{j}$. The one-forms $\omega^{i}$ can be chosen in such a way that

$$
\mathrm{d} \omega^{i}=\frac{1}{2} c_{j k}^{i} \omega^{j} \wedge \omega^{k},
$$

where $\mathrm{d} \omega^{i}$ denotes the exterior differential of the one-form $\omega^{i}$.

In the study of a particular cosmological model of this class, one starts by selecting the structure constants $c_{i j}^{k}$ [which have to satisfy the relations (1)], and then one looks for the one-forms satisfying Eq. (2), in order to construct the space-time metric. As we shall show in Sec. 3.1, starting from a solution of Eq. (2), with specific values of the structure constants, expressed with the aid of the imaginary unit $i$, one can readily construct solutions for other values of the structure constants by simply replacing i by j or $\varepsilon$. In Sec. 3.2, we solve Eq. (2) simultaneously for six different Bianchi types, making use of structure constants that depend on a parameter that may take the values $\mathrm{i}, \mathrm{j}$ or $\varepsilon$.

\subsection{Obtaining two Bianchi types from one}

In this subsection we make use of a solution of Eq. (2) for the structure constants $c_{j k}^{i}=\varepsilon_{i j k}$ and use it to find the solution of (2) for other values of the structure constants.

One can readily verify that the one-forms $\omega^{1}, \omega^{2}, \omega^{3}$, given by

$$
\begin{aligned}
& \omega^{1}=\cos \psi \mathrm{d} \theta+\sin \psi \sin \theta \mathrm{d} \phi, \\
& \omega^{2}=\sin \psi \mathrm{d} \theta-\cos \psi \sin \theta \mathrm{d} \phi, \\
& \omega^{3}=\mathrm{d} \psi+\cos \theta \mathrm{d} \phi,
\end{aligned}
$$

where $\phi, \theta, \psi$ is a local coordinate system, satisfy the relations (2) with $c_{j k}^{i}=\varepsilon_{i j k}$, corresponding to the type IX algebra in the Bianchi classification (see, e.g., Ref. [3], Exercise [26.2]). Equations (3) are equivalent to

$$
\begin{aligned}
\omega^{1}+\mathrm{i} \omega^{2} & =\mathrm{e}^{\mathrm{i} \psi}(\mathrm{d} \theta-\mathrm{i} \sin \theta \mathrm{d} \phi), \\
\omega^{3} & =\mathrm{d} \psi+\cos \theta \mathrm{d} \phi,
\end{aligned}
$$

and if we replace the imaginary unit $\mathrm{i}$ appearing in Eqs. (4) by the unit h, which can be $\mathrm{i}, \mathrm{j}$, or $\varepsilon$, we obtain

$$
\begin{aligned}
\omega^{1}+\mathrm{h} \omega^{2} & =\mathrm{e}^{\mathrm{h} \psi}(\mathrm{d} \theta-\mathrm{h} \sin \theta \mathrm{d} \phi), \\
\omega^{3} & =\mathrm{d} \psi+\cos \theta \mathrm{d} \phi,
\end{aligned}
$$

which define new sets of one-forms (which we shall denote by the same symbols $\omega^{i}$ ), depending on the choice of h. In fact, a straightforward computation shows that

$$
\mathrm{d}\left(\omega^{1}+\mathrm{h} \omega^{2}\right)=\mathrm{h} \omega^{3} \wedge\left(\omega^{1}+\mathrm{h} \omega^{2}\right), \quad \mathrm{d} \omega^{3}=\omega^{1} \wedge \omega^{2} .
$$

Separating the real and imaginary parts of the first of these equations (assuming that the $\omega^{i}$ are real) we see that the oneforms $\omega^{i}$ defined by Eqs. (5) satisfy Eqs. (2) with

$$
c_{23}^{1}=-\mathrm{h}^{2}, \quad c_{31}^{2}=1, \quad c_{12}^{3}=1 .
$$

The other structure constants are equal to zero or are obtained from those given in Eq. (6) making use of the antisymmetry of the structure constants in the two subscripts. (Note that, in all cases, these constants are real.)

When $\mathrm{h}=\mathrm{j}\left(\right.$ i.e., $\mathrm{h}^{2}=1$ ) the structure constants (6) correspond to the type VIII algebra in the Bianchi classification, and (using the fact that $\mathrm{e}^{\mathrm{j} \psi}=\cosh \psi+\mathrm{j} \sinh \psi$ ) Eqs. (5) give

$$
\begin{aligned}
& \omega^{1}=\cosh \psi \mathrm{d} \theta-\sinh \psi \sin \theta \mathrm{d} \phi, \\
& \omega^{2}=\sinh \psi \mathrm{d} \theta-\cosh \psi \sin \theta \mathrm{d} \phi, \\
& \omega^{3}=\mathrm{d} \psi+\cos \theta \mathrm{d} \phi
\end{aligned}
$$

[cf. Eqs. (3)].

When $\mathrm{h}=\varepsilon$ the structure constants (6) correspond to the type $\mathrm{VII}_{0}$ algebra and Eqs. (5) amount to (note that $\mathrm{e}^{\varepsilon \psi}=1+\varepsilon \psi$ )

$$
\begin{aligned}
& \omega^{1}=\mathrm{d} \theta, \quad \omega^{2}=\psi \mathrm{d} \theta-\sin \theta \mathrm{d} \phi, \\
& \omega^{3}=\mathrm{d} \psi+\cos \theta \mathrm{d} \phi
\end{aligned}
$$

[cf. Eqs. (3) and (7)] and one can readily verify that these one-forms satisfy

$$
\mathrm{d} \omega^{1}=0, \quad \mathrm{~d} \omega^{2}=\omega^{3} \wedge \omega^{1}, \quad \mathrm{~d} \omega^{3}=\omega^{1} \wedge \omega^{2},
$$

in agreement with Eqs. (6), for $h=\varepsilon$. It should be remarked that Eqs. (7) and (8) have been obtained without making use of changes of coordinates or limiting processes.

The three Lie algebras considered in this subsection are the Lie algebras of the dynamical symmetry groups found in Ref. [1] and they correspond to the rotation group $\mathrm{SO}(3)$, the Lorentz group in two spatial dimensions, and the group of rigid motions of the Euclidean plane.

\subsection{Direct integration. Six Bianchi types for the price of one}

In this subsection, we follow a direct approach specifying a family of structure constants, similar to (6), and looking for solutions of Eqs. (2).

We shall consider the family of structure constants determined by

$$
c_{12}^{2}=-K, \quad c_{31}^{2}=-\mathrm{h}^{2}, \quad c_{12}^{3}=1, \quad c_{31}^{3}=K,
$$

where $K$ is a real constant and, again, h can be the unit i,j or $\varepsilon$. Depending on the choice of $h$ and $K$, we have six different 
Bianchi types; if $K=0$, they belong to the class A (characterized by the condition $c_{i j}^{i}=0$ ) and if $K \neq 0$, they belong to the class B (with $c_{i j}^{i} \neq 0$ ).

Substituting the values (10) into Eq. (2) we have the explicit expressions

$$
\begin{aligned}
& \mathrm{d} \omega^{1}=0, \quad \mathrm{~d} \omega^{2}=-K \omega^{1} \wedge \omega^{2}-h^{2} \omega^{3} \wedge \omega^{1}, \\
& \mathrm{~d} \omega^{3}=\omega^{1} \wedge \omega^{2}+K \omega^{3} \wedge \omega^{1} .
\end{aligned}
$$

The first of these equations is equivalent to the existence of a function $x$ such that $\omega^{1}=\mathrm{d} x$. The remaining two equations in (11) are equivalent to the single equation

$$
\begin{aligned}
\mathrm{d}\left(\omega^{2}+\mathrm{h} \omega^{3}\right) & =(\mathrm{h}-K) \omega^{1} \wedge\left(\omega^{2}+\mathrm{h} \omega^{3}\right) \\
& =(\mathrm{h}-K) \mathrm{d} x \wedge\left(\omega^{2}+\mathrm{h} \omega^{3}\right),
\end{aligned}
$$

hence, $\mathrm{d}\left[\mathrm{e}^{-(\mathrm{h}-K) x}\left(\omega^{2}+\mathrm{h} \omega^{3}\right)\right]=0$, which, in turn, is equivalent to the existence of two real-valued functions, $y$ and $z$, such that

$$
\begin{aligned}
\omega^{2}+\mathrm{h} \omega^{3} & =\mathrm{e}^{(\mathrm{h}-K) x}(\mathrm{~d} y+\mathrm{hd} z) \\
& =\mathrm{e}^{\mathrm{h} x} \mathrm{e}^{-K x}(\mathrm{~d} y+\mathrm{hd} z) .
\end{aligned}
$$

Separating the real and imaginary parts of Eq. (12) we obtain the expressions for $\omega^{2}$ and $\omega^{3}$.

When $\mathrm{h}=\mathrm{i}$, from Eqs. (12) we obtain

$$
\begin{aligned}
& \omega^{1}=\mathrm{d} x, \quad \omega^{2}=\mathrm{e}^{-K x} \cos x \mathrm{~d} y-\mathrm{e}^{-K x} \sin x \mathrm{~d} z, \\
& \omega^{3}=\mathrm{e}^{-K x} \sin x \mathrm{~d} y+\mathrm{e}^{-K x} \cos x \mathrm{~d} z .
\end{aligned}
$$

Equations (10) show that, if $K=0$, these one-forms correspond to the type $\mathrm{VII}_{0}$ algebra in the Bianchi classification and, if $K \neq 0$, they correspond to the type VII algebra. The one-forms (8) also correspond to this algebra; in fact, the coordinate transformation $x=\theta, y=\psi \sin \theta, z=\phi+\psi \cos \theta$ shows the equivalence of the two results.

Similarly, when $\mathrm{h}=\mathrm{j}$, Eqs. (12) yield

$$
\begin{aligned}
& \omega^{1}=\mathrm{d} x, \quad \omega^{2}=\mathrm{e}^{-K x} \cosh x \mathrm{~d} y+\mathrm{e}^{-K x} \sinh x \mathrm{~d} z, \\
& \omega^{3}=\mathrm{e}^{-K x} \sinh x \mathrm{~d} y+\mathrm{e}^{-K x} \cosh x \mathrm{~d} z,
\end{aligned}
$$

which correspond to the type $\mathrm{VI}_{0}$ algebra if $K=0$, and to the type VI algebra if $K \neq 0$. (When $K$ has the specific value 1 , we obtain the type III algebra.)

Finally, when $\mathrm{h}=\varepsilon$, from Eqs. (12) we have

$$
\omega^{1}=\mathrm{d} x, \quad \omega^{2}=\mathrm{e}^{-K x} \mathrm{~d} y, \quad \omega^{3}=\mathrm{e}^{-K x}(x \mathrm{~d} y+\mathrm{d} z) .
$$

When $K=0$ these forms correspond to the type II algebra and, if $K \neq 0$, to the type IV algebra.

\subsection{Two isolated types}

There are two Bianchi types not included in the results above: the types I and V. The type I correspond to $c_{j k}^{i}=0$ and the solution of Eqs. (2) is trivially given by $\omega^{1}=\mathrm{d} x, \omega^{2}=\mathrm{d} y$, $\omega^{3}=\mathrm{d} z$, where $x, y, z$ are real-valued functions.
For the type V, the structure constants can be chosen as

$$
c_{12}^{2}=-1, \quad c_{31}^{3}=1 .
$$

Then Eqs. (2) give $\mathrm{d} \omega^{1}=0, \mathrm{~d} \omega^{2}=-\omega^{1} \wedge \omega^{2}, \mathrm{~d} \omega^{3}=$ $-\omega^{1} \wedge \omega^{3}$. The first of these equations leads to $\omega^{1}=\mathrm{d} x$, where $x$ is some real-valued function, and the last two equations can be combined in the form

$$
\begin{aligned}
\mathrm{d}\left(\omega^{2}+\mathrm{h} \omega^{3}\right) & =-\omega^{1} \wedge\left(\omega^{2}+\mathrm{h} \omega^{3}\right) \\
& =-\mathrm{d} x \wedge\left(\omega^{2}+\mathrm{h} \omega^{3}\right),
\end{aligned}
$$

which leads to

$$
\omega^{2}+\mathrm{h} \omega^{3}=\mathrm{e}^{-x}(\mathrm{~d} y+\mathrm{hd} z)
$$

and this is equivalent to $\omega^{2}=\mathrm{e}^{-x} \mathrm{~d} y, \omega^{3}=\mathrm{e}^{-x} \mathrm{~d} z$, regardless of the choice for $h$.

It may be noticed that in the case of the type I we can write $\omega^{2}+\mathrm{h} \omega^{3}=\mathrm{d} y+\mathrm{hd} z$, but this leads to the expressions given above, no matter how we choose $h$.

\section{Lie groups}

The vector fields $\mathbf{X}_{i}$ mentioned at the beginning of Sec. 3 define a Lie algebra and therefore can be regarded as leftinvariant vector fields on the manifold of a Lie group generated by this Lie algebra (which is defined up to homomorphism) (see, e.g., Refs. [7, 8]). The one-forms $\omega^{i}$ are then a basis for the right-invariant one-forms on the group manifold (and Eqs. (2) are essentially the Maurer-Cartan equations). The explicit expression for the one-forms in terms of coordinates and the fact that they are right-invariant allow us to find the group operation, without making use of the exponential map (see, e.g., Ref. [8], chap. 7).

For instance, making use of the one-forms (15), with $K=0$, one finds that, in terms of the coordinates $x, y, z$, the group operation is locally given by $x\left(g g^{\prime}\right)=x(g)+x\left(g^{\prime}\right)$, $y\left(g g^{\prime}\right)=y(g)+y\left(g^{\prime}\right), z\left(g g^{\prime}\right)=z(g)+z\left(g^{\prime}\right)-y(g) x\left(g^{\prime}\right)$, for any pair of elements of the group, $g, g^{\prime}$. One can verify that these formulas can be reproduced by associating to an element $g$ of the group, the $3 \times 3$ matrix

$$
\left(\begin{array}{ccc}
1 & y & -z \\
0 & 1 & x \\
0 & 0 & 1
\end{array}\right) .
$$

\section{Final remarks}

The results of Sec. 3 show that, at least in the examples considered here, the nonlinear appearance of the unit $h$ in Eqs. (5) and (12) leads to nonequivalent solutions of Eqs. (2). It would be interesting to know in which other cases a similar trick may produce effortlessly new solutions from a given one by means of the use of the complex, double, and dual numbers. 
1. G. F. Torres del Castillo, Some applications in classical mechanics of the double and the dual numbers, Rev. Mex Fis. 65 (2019) 152, https: //doi.org/10.31349/ RevMexFisE.65.152

2. V. K. Kisil, Geometry of Möbius Transformations (Imperial College Press, London, 2012), Chap. 3, https://doi. org/10.1142/p835

3. L. P. Hughston and K. P. Tod, An Introduction to General Relativity (Cambridge University Press, Cambridge, 1990), Chap. 26, https://doi.org/10.1017/ CB09781139171977

4. H. Stephani, D. Kramer, M. MacCallum, C. Hoenselaers, and E. Herlt, Exact Solutions of Einstein's Field Equations (Cambridge University Press, Cambridge, 2003), Chap. 8, https : //doi.org/10.1017/CB09780511535185.
5. Ø. Grøn and S. Hervik, Einstein's General Theory of Relativity (Springer-Verlag, New York, 2007), Chap. 15, http: //doi.org/10.1007/978-0-387-69200-5

6. G. F. R. Ellis, R. Maartens, and M. A. H. MacCallum, Relativistic Cosmology (Cambridge University Press, Cambridge, 2012), Sec. 18.3, https://doi.org/10.1017/ CB09781139014403.

7. D. H. Sattinger and O. L. Weaver, Lie Groups and Algebras with Applications to Physics, Geometry, and Mechanics (Springer-Verlag, New York, 1986), Chaps. 7, 8, http: //doi.org/10.1007/978-1-4757-1910-9

8. G. F. Torres del Castillo, Differentiable Manifolds: A Theoretical Physics Approach, 2nd ed. (Birkhäuser, New York, 2020). http://doi.org/10.1007/978-3-030-45193-6. 